\title{
Haemovascular changes in septic shock
}

\author{
A. LitToN \\ Southern General Hospital, Glasgow
}

\section{Summary}

In refractory hypotension of doubtful origin, bacterial factors should be suspected and blood cultures taken. Gram-negative organisms accounted for the majority of cases of bacteraemia.

Unlike haemorrhagic shock, the blood volume estimated in patients in shock associated with infection was not found to be reduced.

In some patients, when vital signs became stabilized, a marked expansion in the vascular capacity was demonstrated.

Plasma of patients in bacteraemic shock was markedly vasoconstrictor.

The metabolic acidosis present in cases of septic shock was due to lactic-acidaemia.

Sepsis can be suspected when thrombocytopenia is associated with leucocytosis.

\section{Introduction}

Infection associated with hypotension has been described from time to time, but it was Waisbren who in 1951 first described bacteraemic shock as a specific clinical entity where hypotension was associated with Gram-negative bacteraemia. Later, Borden \& Hall (1951) and Braude et al. (1953) suggested that it was endotoxin from Gram-negative bacteria which was the cause of the toxaemia and shock.

Since the introduction and widespread use of antibiotic therapy in hospital, certain bacterial species previously considered to have little pathogenicity for man are now being recognized as the organisms responsible for severe and sometimes fatal infections. At present, the more important pathogens are the Gram-negative bacilli of the Escherichia, KlebsiellaAerobacter, Proteus, Pseudomonas and Bacteroides genera, many of which are among the normal bacterial commensals of man.

\section{Positive blood cultures}

In cases of hypotension where the cause is not obvious bacterial causes may readily be overlooked unless blood cultures and other bacterial specimens are taken. Table 1 demonstrates the bacteria isolated from blood cultures of patients in shock where a bacterial aetiology was suspected.
TABLE 1. Bacteria isolated from blood cultures of patients in bacteraemic shock

\begin{tabular}{lr}
\hline $\begin{array}{l}\text { Organisms recovered } \\
\text { from blood cultures }\end{array}$ & No. \\
\hline Staphylococcus aureus & 2 \\
Escherichia coli & 13 \\
Klebsiella-Aerobacter & 6 \\
Pseudomonas & 5 \\
Proteus & 2 \\
Bacteroides & 2 \\
Alcaligenes faecalis & 2 \\
Serratia & 1 \\
Monilia & 1 \\
\hline
\end{tabular}

Although staphylococcal infections are still important, in the surgical patients studied Gramnegative organisms were the dominant bacteria isolated from blood cultures in established bacteraemia. Escherichia coli was the most frequent species isolated from the blood.

\section{Blood volume studies}

Although different types of shock are described, e.g. cardiac, haemorrhagic and bacteraemic, patients with low flow states often present complicated clinical problems with many contributing factors. Blood volume estimations were made in a series of patients in shock where the refractory hypotension was associated with infection as the evident clinical cause and where blood cultures were later found to be positive. Plasma volumes were estimated using Evans Blue or ${ }^{131}$ I-labelled albumin. From the venous haematocrits (corrected for whole body haematocrit) total blood volumes were calculated. Occasionally the red cell mass was estimated independently by labelling the patient's own red cells with radiochromate $\left({ }^{51} \mathrm{Cr}\right)$ and retransfusing the autogenous isotope-labelled cells.

Fig. 1 shows the blood volume results of those patients in bacteraemic shock. The observed results were plotted against the expected blood volumes which were obtained from a nomogram giving the blood volume as a function of the patient's sex, age and weight. In septic shock the observed blood volume did not appear to be lower than the expected value. On the contrary there appeared to be an expansion of the vascular space-the effective blood volume 


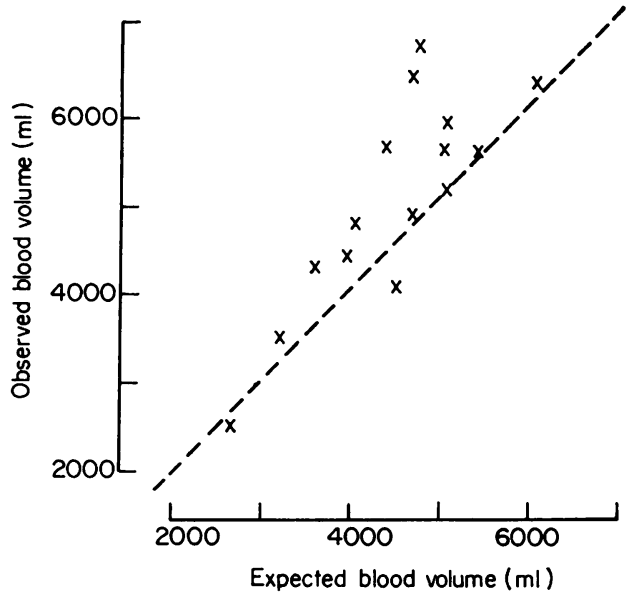

FIG. 1. Blood volumes in fifteen patients with hypotension associated with bacteraemia. Comparison of the expected blood volume with the observed blodd volume. The line of coincidence indicates that hypovolaemia is not a major factor in 'septic shock'.

required to maintain an adequate arterial blood pressure was often considerably in excess of the expected normal value.

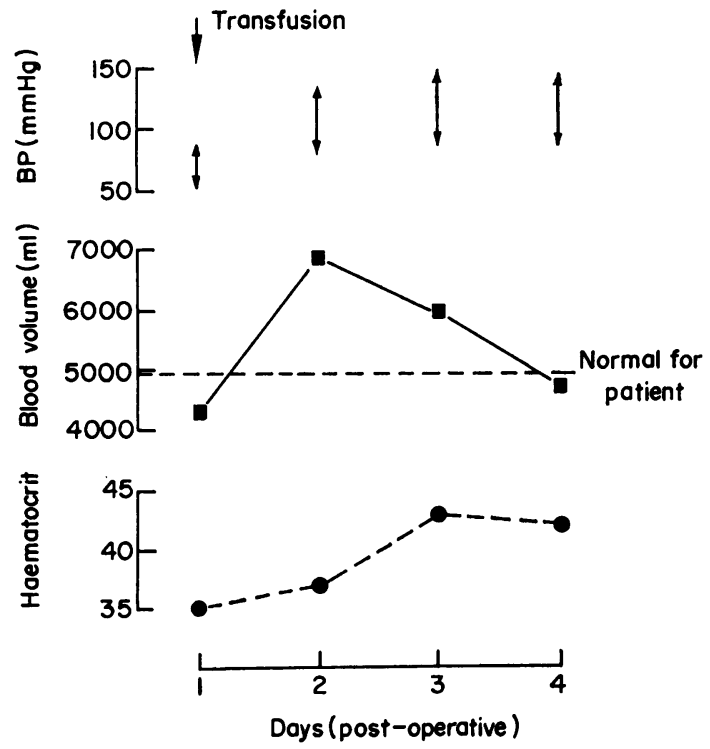

FIG. 2. Serial blood volume, arterial blood pressure and haematocrit data from a patient in shock associated with $E$. coli bacteraemia complicating peritonitis.

Fig. 2 shows the changes in the blood volume of a patient in shock associated with generalized peritonitis. Intravenous fluids were given in an attempt to restore systemic arterial blood pressure without causing undue elevation of the central venous pres- sure. The following day when the systemic arterial blood pressure was stable, the blood volume had expanded $40 \%$ above the expected normal. In the course of the following 5 days as clinical improvement progressed the blood volume fell to the expected normal for the patient. The central venous pressure had been monitored throughout the period of hypotension and had never exceeded $15 \mathrm{~cm}$ water.

Blood volume studies are valuable in shock but the results must always be considered jointly with other measurements, e.g. arterial and venous pressures, pulse rate, and cardiac and pulmonary findings.

It is frequently not possible to assess accurately the expected blood volume in critically ill patients and, as in the case described, the effective circulating blood volume may be considerably in excess of the normal level.

\section{Vasoactive properties of plasma}

In addition to volume changes, the vasoactivity of the plasma of patients in bacteraemic shock was studied. Using the hamster cheek pouch as a model to study the vasoactivity of plasma (Litton, 1968) all patients in shock associated with sepsis demonstrated marked vasoconstrictor properties in their plasma (Fig. 3).

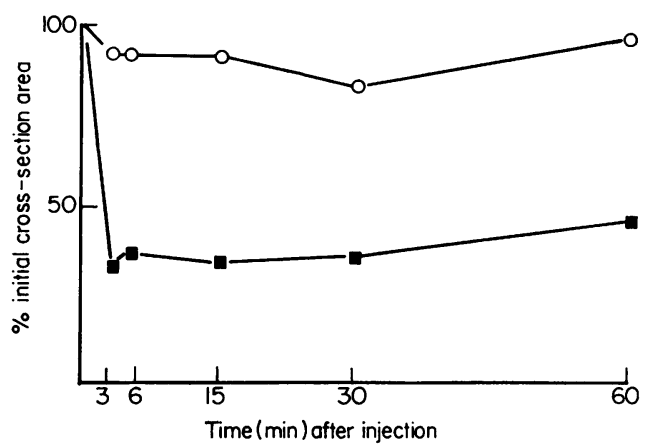

FIG. 3. The vasoactivity of plasma $(0.05 \mathrm{ml})$ of patients in bacteraemic shock on the microvasculature of the hamster cheek pouch. $O$, Veins; $\square$, arteries.

Renal vasoconstriction with disturbed renal function was common in patients in bacteraemic shock. Oliguria or anuria was associated with elevated blood urea and high serum potassium levels. Serum sodium and chloride levels were usually normal or low.

\section{Anaerobic metabolism and lactic acidosis}

With peripheral vasoconstriction and low flow states tissue hypoxia enforces anaerobic metabolism with the formation of lactate rather than the utilization of pyruvate into the normal Krebs cycle.

Blood lactate and pyruvate levels, $\mathrm{pH}, \mathrm{PCO}_{2}$ and base-excess data were obtained from patients in 


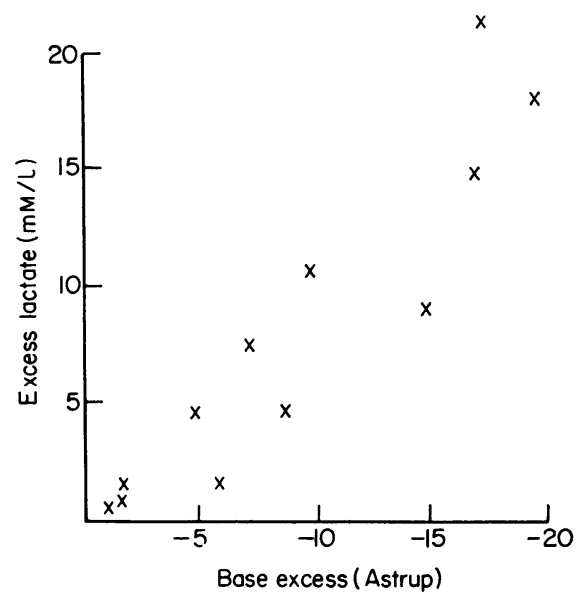

Fig. 4. The correlation of excess lactate and base excess in patients with hypotension associated with sepsis. $r=-0.88$.

shock associated with sepsis. Fig. 4 illustrates the relationship of excess lactate (Huckabee, 1958) to the base excess (Siggaard-Andersen, 1962) in patients in bacterial shock.

The high degree of correlation between the base deficit ard the excess lactate $(P<0.001)$ suggests that the acidosis could be accounted for almost exclusively by the increased concentration of the blood lactate.

\section{Blood coagulation problems}

In septic shock upsets in the blood coagulation mechanism are frequently observed. Hardaway (1962) showed a diphasic response in shock. At first there is hypercoagulability followed by a phase of hypocoagulability and fibrinolysis.

Intravascular clotting has been demonstrated in the microcirculation in shock (McKay \& Shapiro, 1958; Spink \& Vick, 1961).
In several patients in the present review where hypotension was associated with severe infections, thrombocytopenia was observed. Platelet counts were under $100,000 / \mathrm{mm}^{3}$, the lowest value being $5000 / \mathrm{mm}^{3}$. In three cases where bone marrow studies were made to investigate the thrombocytopenia, megakaryocytes were plentiful and in one case where an isologous isotope-tagged platelet infusion was given, sequestration or destruction of platelets appeared to be the main cause of the platelet depletion. At the time of study all patients exhibited a marked polymorphonuclear leucocytosis. There was a striking divergence of platelet and leucocyte counts. Thrombocytopenia associated with leucocytosis in a patient with refractory hypotension usually indicates Gram-negative sepsis.

\section{References}

Borden, C.W. \& HALl, W.H. (1951) Fatal transfusion reactions from massive bacterial contamination of blood. New Engl. J. Med. 245, 760.

Braude, A.I., Siemienski, J., Williams, D. \& Sanford, J.P. (1953) Overwhelming bacteremic shock produced by Gram-negative bacilli: a report of four cases with one recovery. Univ. Mich. med. Bull. 19, 23.

HARDAWAY, R.M. (1962) The role of intravascular clotting in the etiology of shock. Ann. Surg. 155, 325.

HuCKABEE, W.E. (1958) Relationships of pyruvate and lactate during anerobic metabolism. II. Exercise and formation of $\mathrm{O}_{2}$ debt. J. clin. Invest. 37, 255.

LitTon, A. (1968) Microvascular effects of vasoactive agents in shock. Brit. J. Surg. 55, 751.

MCKAY, D.G. \& SHAPIRO, S.S. (1958) Alterations in the blood coagulation system induced by bacterial endotoxins. I. In vivo. Generalised Shwartzman reaction. J. exp. Med. $107,353$.

SiggaARD-Andersen, O. (1962) The pH-log $\mathrm{PcO}_{2}$ blood acid-base nomogram revised. Scand. J. clin. Lab. Invest. 14, 298.

SPINK, W.W. \& VICK, J.A. (1961) Endotoxin shock and the coagulation mechanism: modification of shock with epsilonamino-caproic acid. Proc. Soc. exp. Biol. (N.Y.), 106, 242.

WAISBREN, B.A. (1951) Bacteremia due to Gram-negative bacilli other than the salmonella. Arch. intern. Med. 88, 467. 